\title{
Viral-Mediated Knockdown of mGluR7 in the Nucleus Accumbens Mediates Excessive Alcohol Drinking and Increased Ethanol-Elicited Conditioned Place Preference in Rats
}

\author{
Amine Bahi*, I \\ 'Department of Anatomy, Faculty of Medicine and Health Sciences, United Arab Emirates University, Al Ain, UAE
}

\begin{abstract}
Whether metabotropic glutamate 7 (mGluR7) -activation enhances or diminishes the reinforcing properties of psychostimulants remains unclear. We have previously shown that systemic mGluR7 activation reduced alcohol consumption and preference as well as locomotorstimulating and rewarding properties of ethanol. In this study, we further examined the contribution of mGluR7 on the effect of ethanol within the nucleus accumbens (NAcc), a neural target for many drugs of abuse. Using short hairpin RNA (shRNA)-expressing lentiviral vectors (LV) to alter locally the activity of mGluR7 in male rats, we have shown that blocking mGluR7 expression increased ethanol consumption and preference in a two-bottle choice drinking paradigm with no effect either on saccharin or on quinine used for taste discrimination. In addition, mGluR7 knockdown increases preference for environments previously paired with low doses of ethanol in the conditioned place preference (CPP) test, as it shifted the dose-response curve for ethanol CPP to the left, indicating alterations in the rewarding effects of alcohol. More importantly, mGluR7 blockade in the dorsal striatum (DS) neither affected ethanol consumption nor ethanol-elicited CPP. These results show that levels of mGluR7 in the NAcc regulate responsiveness to alcohol. Taken together, these findings clearly demonstrate that mGluR7 signaling within the NAcc is a key modulator of functional responses to ethanol and offer an important target for regulating the addictive effects of alcohol.

Neuropsychopharmacology (2013) 38, 2 I09-21 19; doi:I0.1038/npp.2012.122; published online II July 2012
\end{abstract}

Keywords: lentivirus; conditioned place preference; mGluR7; ethanol; shRNA; two-bottle choice

\section{INTRODUCTION}

Although alcoholism is one of the leading causes of disease and premature deaths in modern western societies, alcohol is a legal drug that is widely consumed in a social context. In addition, while ethanol is considered the major reinforcing component in alcoholic beverages responsible for alcohol abuse, the mechanisms of ethanol addiction are not completely known. Understanding the neural and molecular mechanisms underlying ethanol addiction is crucial for the development of effective therapies for alcoholism. It is currently believed that the nucleus accumbens (NAcc) is critically involved in many drug-related behaviors (eg, (Badanich et al, 2006; Dackis and O'Brien, 2001; Di Chiara et al, 1993; $\mathrm{Li}$ and Kauer, 2004; Wenger et al, 2003)). It is well established that the central glutamate system is involved in significant neuro-adaptive changes in response to ethanol exposure (Flatscher-Bader et al, 2008; Kemppainen et al,

\footnotetext{
*Correspondence: Dr A Bahi, Department of Anatomy, Faculty of Medicine \& Health Sciences, Tawam Medical Campus, United Arab Emirates University, PO Box 17666, Al Ain, UAE, Tel: +971 3 7137 516, Fax: +97I 37672 033, E-mail: amine.bahi@uaeu.ac.ae Received 30 January 2012; revised 13 June 2012; accepted I4 June 2012
}

2010; Wills et al, 2012; Xiao et al, 2009). Thus, targeting the glutamatergic transmission may provide important therapeutic targets for investigation of exposure to alcohol (De Witte et al, 2005; Pelc, 1997).

L-glutamate is the most abundant excitatory amino-acid neurotransmitter in the mammalian central nervous system. There are two types of glutamate receptors: metabotropic and ionotropic receptors (for review, see (Bird and Lawrence, 2009; Olive, 2009; Uys and LaLumiere, 2008)). More evidence regarding the involvement of the glutamatergic system in ethanol addiction focuses on the metabotropic receptors (mGluR). In fact, there is increasing evidence that group III mGluRs, particularly mGluR7 may be implicated in a number of psychiatric disorders (O'Connor et al, 2010).

The mGluR7 is a G-protein-coupled receptor widely expressed in the brain, with the highest levels of mGluR7 mRNA present in emotion-related regions, including the prefrontal cortex, hippocampus, striatum, amygdala and thalamus (Kinoshita et al, 1998; Okamoto et al, 1994). As such, dysregulation of mGluR7 has been linked to many psychiatric diseases including depression (Bradley et al, 2011; Cryan et al, 2003), anxiety (Fendt et al, 2008; Mitsukawa et al, 2006), schizophrenia (Bolonna et al, 2001; Ohtsuki et al, 2008) and drug addiction (Bahi et al, 
2012; Li et al, 2009, 2010). In rodents, results have indicated that the mGluR7 allosteric agonist $N, N$-dibenzyhydryl-ethane1,2-diamine dihydrochloride (AMN082) dose dependently inhibits cocaine-induced enhancement of electrical brainstimulation reward and intravenous cocaine self-administration in rats (Li et al, 2009). In the same study, local infusions of AMN082 in the NAcc, but not in the dorsal striatum (DS), dose-dependently inhibited cocaine self-administration. This effect was reversed when rats were pre-injected with the mGluR7-selective allosteric antagonist 6-(4-methoxyphenyl)-5-methyl-3-pyridin-4-ylisoxazolo[4,5c]pyridin-4(5H)-one (MMPIP) (Li et al, 2009). The same group of researchers has also shown that systemic as well as intra-NAcc injection of AMN082 dose dependently inhibits cocaine-induced reinstatement of drug-seeking behavior after extinction ( $\mathrm{Li}$ et al, 2010). With regard to ethanol addiction, our findings have documented that, in rats, $10 \mathrm{mg} / \mathrm{kg}$ AMN082 significantly decreased voluntary ethanol consumption and preference in a two-bottle choice drinking paradigm (Bahi et al, 2012). This effect was blocked when MMPIP (10 mg/kg) was co-administered with AMN082. More recently, we have reported that mGluR7 activation using AMN082 had no effect on ethanol-induced conditioned place preference (CPP) extinction (Bahi, 2012) but significantly reduced reinstatement after priming with a single dose of ethanol, an effect reversed by co-administration of MMPIP (Bahi, 2012). In the light of these findings, Gyetvai et al (2011) have shown that sub-congenic, and congenic mice carrying an mGluR7 variant characterized by higher mGluR7 mRNA drink less alcohol, in contrast to mGluR7 knockout mice, which express increased voluntary alcohol consumption.

Most of the studies cited above involved systemic injection of mGluR7 pharmacological agents or constitutive transgenic knockout mice. Therefore, we have decided to employ a technique of viral-mediated gene transfer to knock down mGluR7 expression using short hairpin RNA (shRNA)-expressing lentiviral vectors (LV) within the NAcc (and the DS) to study the effects of reduced mGluR7 mRNA levels on ethanol-related behaviors. This technique was extensively used in our laboratory to manipulate a number of genes in different CNS regions of both rats and mice (Bahi et al, 2005, 2008a,b; Bahi and Dreyer, 2012a,b).

The present study was designed to clarify the role of NAcc mGlu7 receptors using LV-mediated gene transfer, a method capable of targeting a specific receptor population in a specific brain region. Based on our previous findings, our current hypothesis is that reduced expression of mGluR7 in NAcc neurons increases the stimulant and rewarding actions of ethanol. To test this hypothesis, we used LV to block the expression of mGlu7 receptor terminals of NAcc neurons. We evaluated the effect of mGluR7 knockdown on the ability of several doses of ethanol to establish CPP. We also measured voluntary alcohol consumption preference in rats using a two-bottle choice drinking paradigm.

\section{MATERIALS AND METHODS}

\section{Animals}

Male Wistar rats (250-300 g) were group housed (five per cage) at a room temperature of approximately $22^{\circ} \mathrm{C}$ with a
$12 \mathrm{~h} / 12 \mathrm{~h}$ light/dark cycle and allowed to adapt to this environment for a period of 7 days before the experiments began. The procedures were approved by the local Animal Research Ethics Committee (Protocols no. A09/11 and A18/11).

\section{Drugs}

For the intraperitoneal (i.p.) injection used in the CPP experiments, $20 \%(v / v)$ ethanol was prepared in isotonic saline $(\mathrm{NaCl} 0.9 \%)$ and the volume of injection was adjusted to body weight. In contrast, for the two bottle choice drinking experiments, ethanol was diluted to $2.5,5,10$ or $20 \%(v / v)$ solution in tap water. For taste discrimination, saccharin (Sigma) was prepared as a 0.035 or $0.07 \%(w / v)$ solution in tap water. Quinine hemisulfate salt (Sigma) was prepared as a 25 or $50 \mu \mathrm{M}$ solution in tap water.

\section{Virus Preparation of mGluR7 shRNA-Expressing Vectors and Viral Delivery}

The cloning of the mGlu7 receptor shRNA-expressing viral vectors was performed as described previously (Bahi et al, 2004a, 2005, 2006). Briefly, a 21-nucleotide mGluR7 shRNA sequence (NCBI Reference Sequence: NM_031040; 1041-1061: $\left(5^{\prime}\right.$-GCTTACTTCACATCCCGGACA- $\left.3^{\prime}\right)$ or the scrambled control shRNA ( $5^{\prime}$-GTTGGCAGCAGTAACGCAGCA- $\left.3^{\prime}\right)$ were chosen. To each oligo, an $\mathrm{XhoI}$ restriction site was added at $3^{\prime}$ and a U6- $3^{\prime}$-specific 15 -mer at $5^{\prime}$. Using the pSilencer 1.0U6 (Ambion, UK) as a template and a U6 promoter-specific forward primer containing a $B a m \mathrm{HI}$ restriction site $\left(5^{\prime}\right.$ GCGGATCCCGCTCTAGAACTAGTGC-3'), each shRNA target was added to the mouse U6 promoter by PCR. The PCR conditions were highly stringent to avoid mutations within the targets. The following PCR program was performed: $180 \mathrm{~s}$ at $94{ }^{\circ} \mathrm{C}$ (initial denaturation) followed by $94{ }^{\circ} \mathrm{C}$ for $30 \mathrm{~s}, 64^{\circ} \mathrm{C}$ for $30 \mathrm{~s}$, and $72{ }^{\circ} \mathrm{C}$ for $50 \mathrm{~s}$, repeated for 40 cycles. The PCR reaction contained $4 \%$ dimethyl sulfoxide (Sigma). The amplicon was then purified, digested with BamHI/XhoI, and ligated into the pTK431 previously digested with the same enzymes. Preparation of LV was initiated by triple transfection of HEK293T cells by a calcium phosphate method using pTK431 together with $\mathrm{p} \Delta \mathrm{NRF}$ and pMDGVSV. Cells were harvested $72 \mathrm{~h}$ later and viruses were concentrated from the supernatant by ultracentrifugation. Vectors were resuspended in PBS-BSA and stored at $-80^{\circ} \mathrm{C}$ until use (Bahi et al, 2008a, 2012; Bahi and Dreyer, 2008, 2012a,b).

Local stereotaxic injections were used to deliver the viruses into the NAcc or DS. Rats were anesthetized with a ketamine/xylazine mixture (100 and $10 \mathrm{mg} / \mathrm{kg}$ respectively, i.p.) and installed in a stereotaxic frame. Using a precision Hamilton micro-syringe with a $26 \mathrm{G}$ needle, rats were bilaterally infused with viral solution using the following coordinates: NAcc (anterior: $+1.7 \mathrm{~mm}$, lateral: $\pm 1 \mathrm{~mm}$, ventral: $7 \mathrm{~mm}$ from the skull), DS (anterior: $+0.48 \mathrm{~mm}$, lateral: $\pm 3 \mathrm{~mm}$, ventral: $5 \mathrm{~mm}$ from the skull) (Paxinos and Watson, 1998). Viruses were infused at a rate of $0.2 \mu \mathrm{l} / \mathrm{min}$ for $5 \mathrm{~min}$ (final volume $1 \mu \mathrm{l} / \mathrm{site}$ ). The Hamilton micro-syringe was held in place for an additional $10 \mathrm{~min}$ before being removed. Following surgery, the rats were allowed to recover for 
10 days before undergoing the behavioral assays (Bahi et al, 2004b, 2008a,b).

\section{Ethanol-Induced CPP}

The procedure for evaluating EtOH-CPP has been described previously (Bahi et al, 2008a,b, 2012). Briefly, the CPP experiments were conducted in a two-chambered place conditioning apparatus connected to an overhead camera. Two $(30 \times 30 \times 30 \mathrm{~cm})$ conditioning chambers, one white with a large grid floor and the other black with a narrow grid floor, were connected by a manually operated guillotine door $(10 \mathrm{~cm})$. The chamber's luminance was adjusted so that the environmental (visual and tactile) cues would not produce a significant baseline preference for a specific chamber. Most rats spent approximately $50 \%$ of the time in each chamber $(p>0.05)$. Therefore, conditioning was performed using an unbiased, balanced protocol.

On day 1, (pre-conditioning) naïve- or ethanol-exposed rats were injected with saline $(1 \mathrm{ml} / \mathrm{kg}$, i.p.) and placed between the two chambers and were allowed to explore freely both compartments for $15 \mathrm{~min}$.

After baseline assessment, the rats were stereotaxically injected with viral vectors as described above and given a 10-day recovery period (days 2-11).

During conditioning (days 12-15), each rat would be injected twice daily (morning and afternoon) with either vehicle or ethanol for 4 days and confined to one of the chambers for $20 \mathrm{~min}$ after injection. During drug conditioning, rats were randomly assigned to receive either saline or ethanol $(0,0.5$ or $1 \mathrm{~g} / \mathrm{kg}$, prepared from $20 \%$ ethanol solution in isotonic saline $v / v$ ). The ethanol- and salinepaired conditioning compartments and the time of the drug or saline conditioning session (morning or afternoon) were counterbalanced across all groups.

On the day following the last conditioning (day 16, postconditioning), an individual rat was injected with saline $(1 \mathrm{ml} / \mathrm{kg}$, i.p.) and placed between the two compartments. The rat had free access to both chambers for $15 \mathrm{~min}$ (CPP test) during which the total time spent in each chamber was recorded. CPP was determined by plotting the time spent in the ethanol-paired compartment during the post-conditioning (day 16).

\section{Twenty-Four-Hour, Two-Bottle Choice Ethanol Consumption and Preference}

The procedures for measuring 24-h ethanol consumption and preference have been described previously (Bahi et al, 2012). Briefly, rats were first acclimated to drinking tap water for a week from two bottles fitted with metal drinking spouts. On day 1 of alcohol access, one water bottle was replaced with a bottle containing $2.5 \%$ ethanol. This ethanol concentration was offered for 3 consecutive days, and then increased to $5 \%$, then $10 \%$, and finally $20 \%$ at 3 -day intervals. The position of the ethanol and water bottles was alternated every day. The rats were weighed every 3 days. The amount of water or ethanol consumed was determined by weighing the bottles before access and after $24 \mathrm{~h}$ of access. Ethanol consumption was determined by calculating the grams of alcohol consumed per $100 \mathrm{~g}$ of body weight. The ethanol preference ratio was calculated by the following formula: Preference ratio $=$ ethanol solution intake/total fluid intake (ethanol solution + water). The total fluid intake was calculated by calculating the volumes of water and ethanol consumed and adjusting to $100 \mathrm{~g}$ of body weight. Potential leakage and evaporation were adjusted by using the average volume lost from identical bottles placed in an empty cage on the same rack as the experimental animals.

\section{Twenty-Four-Hour, Two-Bottle Choice Saccharin and Quinine Consumption and Preference}

Saccharin and quinine consumption and preference were measured in the same rats 1 week after measurement of baseline ethanol drinking. During the intervening week, the animals were left undisturbed with ad libitum water access. Methods were as described for ethanol drinking and two concentrations of each tastant were evaluated (saccharin: 0.035 and $0.07 \%$; quinine: 25 and $50 \mu \mathrm{M}$ ) in ascending and counterbalanced order, consistent with our previous similar studies (Bahi et al, 2012).

\section{Total RNA Isolation and cDNA Quantification Using RT-PCR}

At the end of the behavioral experiments, animal's mGluR7 transcripts were quantified by mean of RT-PCR. Total RNA from brain tissue (NAcc and DS were punched out) were isolated using Trizol (Invitrogen) and reverse transcribed using SuperScript III reverse transcription (Invitrogen). RNA expression for mGluR7 was analyzed by RT-PCR using SyberGreen with temperature cycling parameters consisting of initial denaturation at $94{ }^{\circ} \mathrm{C}$ for $5 \mathrm{~min}$ followed by 40 cycles of denaturation at $94{ }^{\circ} \mathrm{C}$ for $30 \mathrm{~s}$, annealing and extension at $65^{\circ} \mathrm{C}$ for $40 \mathrm{~s}$. PCR for the control gene, glyceraldehyde-3-phosphate dehydrogenase (GAPDH), was run with the same cycling parameters. PCR products were normalized to GAPDH as indicated in the figure legends. The following sets of primers were used for RT-PCR: (mGluR7-Left: 5'-TCTTGGACACTCCCAACTCC-3'; mGluR7Right: $5^{\prime}$ - ATCTTCGTGCTGATGCAGTG- $3^{\prime}$ ) and (GAPDHLeft: 5'-AGACAGCCGCATCTTCTTGT-3'; GAPDH-Right: 5'-CTTGCCGTGGGTAGAGTCAT-3').

\section{Determination of Blood Ethanol Concentration (BEC)}

The procedure for evaluating BEC has been described previously (Bahi et al, 2012). In brief, rats were given an i.p. injection with a $20 \%(v / v)$ solution of ethanol $(2.5 \mathrm{~g} / \mathrm{kg}$ of body weight) in isotonic sterile saline. Tail blood samples were collected in heparinized capillary tubes at $30 \mathrm{~min}$ and again at additional 60-min intervals following injection. A $150-\mu$ l blood sample was drawn at each time point after injection of ethanol. The BEC determinations were made using the NAD-ADH enzymatic assay (Sigma). BEC was measured using a spectrophotometric method based on the conversion of ethanol to acetaldehyde by alcohol dehydrogenase (Keiver et al, 2005). The concentration of ethanol in the blood samples was calculated using an ethanol standard curve. 


\section{Statistical Analysis}

For statistical comparisons, the software package SPSS (version 19.0) was used. All data were expressed as means \pm SEM. Voluntary ethanol or tastant consumption, preference ratio and total volume intake were analyzed using two-way analysis of variance (ANOVA) with repeated measures (virus group $\times$ ethanol/tastant concentration). Ethanol-CPP behavior was determined by comparing times spent in the ethanol-paired chamber between pre- and post-test in the same rat. Paired $t$-tests were used to analyze CPP data statistically. BEC data were analyzed using two-way ANOVAs repeated measures (virus group $\times$ time). Post hoc individual mean comparisons were performed with the Bonferroni's test when $F$ values were significant. The level of statistical significance was set at $p<0.05$ at all times.

\section{RESULTS}

GR7-shRNA in the NAcc, but not in the DS, Increased Ethanol-Induced CPP

The rats were first assessed for their baseline preference as described above. Animals were then randomly assigned to two groups: GR7-scr or GR7-shRNA. After stereotaxic surgery, the rats were left to recover for 10 days and trained in the standard ethanol-CPP protocol using $0,0.5$ or $1 \mathrm{~g} / \mathrm{kg}$ ethanol (i.p.). When saline was used for conditioning neither GR7-scr nor GR7-shRNA failed to produce a place preference or aversion, indicating a lack of intrinsic rewarding or aversive effects of these shRNA-expressing viral vectors. The effects of NAcc lentiviral-injections on ethanol-CPP $(0,0.5$, and $1 \mathrm{~g} / \mathrm{kg})$ are shown in Figure $1 \mathrm{a}$. Two-way ANOVA analysis has shown that time spent in the ethanol-paired box varied as a function of virus $\left(\mathrm{F}_{(1,47)}=\right.$ 4.602, $p=0.038)$ and by ethanol concentration $\left(\mathrm{F}_{(2,47)}=\right.$ 22.703, $p<0.001)$. More importantly, the interaction between the viral injection and ethanol concentration was significant: $\left.\mathrm{F}_{(2,47)}=3.139, p=0.054\right)$. Rats receiving viral injections (GR7-scr ( $n=7-9$ /group), GR7-shRNA ( $n=8-9$ / group)) and conditioned to saline did not show preferences for either of the compartments. In contrast, rats receiving GR7-shRNA microinjections into the NAcc spent significantly more time in environments paired with moderate dose $(0.5 \mathrm{~g} / \mathrm{kg})$ of ethanol $(p<0.05$ vs saline $)$, whereas rats receiving GR7-scr microinjections did not prefer the ethanol-paired environment at the same dose $(p<0.05 v s$ GR7-shRNA). When conditioned with a higher dose of ethanol $(1 \mathrm{~g} / \mathrm{kg})$ there was no significant difference between the two groups $(p>0.05)$. These data suggest that NAcc mGluR7 is involved in the reinforcing properties of ethanol.

The second cohort of animals were stereotaxically injected with GR7-scr or GR7-shRNA in the DS and, after recovery, the rats were trained in the standard ethanol-CPP protocol as described above. As shown in Figure 1b, both groups equally exhibited a preference for the ethanol-paired chamber at higher dose $(1 \mathrm{~g} / \mathrm{kg})$ indicating that the reinforcing properties of ethanol are unaffected in the GR7-shRNA rats. Two-way ANOVA analysis has shown that time spent in the ethanol-paired box varied as a function of ethanol concentration $\left(\mathrm{F}_{(2,51)}=34.929, p<0.001\right)$ but viral injection had no main effect $\left(\mathrm{F}_{(1,51)}=0.017, p=0.896\right)$, as well as their
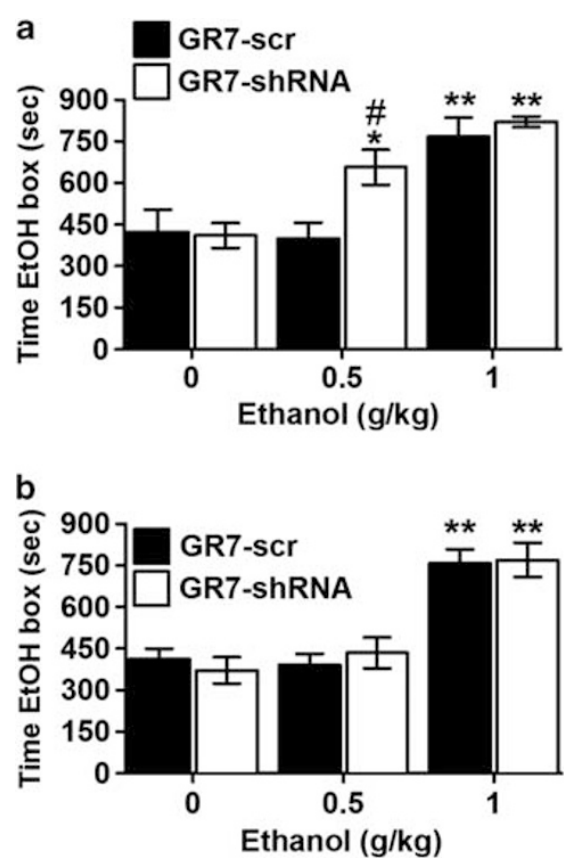

Figure I mGluR7 levels in the nucleus accumbens (NAcc), but not in the dorsal striatum (DS), regulate ethanol-mediated place preference conditioning. (a) GR7-short hairpin RNA (shRNA) expression in the NAcc enhanced sensitivity to $0.5 \mathrm{~g} / \mathrm{kg}$ of ethanol whereas expression of GR7-scr resulted in place preference at the I g/kg of ethanol. (b) GR7-scr and GR7shRNA expression in the DS enhanced sensitivity only to $1 \mathrm{~g} / \mathrm{kg}$ of ethanol. Data are presented as the mean \pm SEM. $* p<0.05$, $* * p<0.01 \mathrm{vs} 0 \mathrm{~g} / \mathrm{kg}$ of ethanol, " $p<0.05$ vs GR7-scr-injected group (two-way analysis of variance, Bonferroni's post hoc test).

interaction (viral injection $\times$ ethanol concentration interaction: $\left.\mathrm{F}_{(2,51)}=0.348, p=0.708\right)$. Rats receiving viral-vector injections in the DS (GR7-scr ( $n=8-9-9 /$ group), GR7shRNA ( $n=8-9-9 /$ group) $)$ and conditioned to saline did not show preferences for either of the compartments. Moreover, both groups of rats did not discriminate between the two compartments when $0.5 \mathrm{~g} / \mathrm{kg}$ ethanol was used for conditioning ( $p>0.05$ vs saline). In contrast, when conditioned with a higher dose of ethanol $(1 \mathrm{~g} / \mathrm{kg})$ there was no significant difference between the two groups $(p>0.05)$ but both groups spent more time in the ethanol-paired chamber $(p<0.05 v s$ saline). These data suggest that when mGluR7 mRNA expression was blocked in the DS, the reinforcing properties of ethanol are intact.

\section{GR7-shRNA in the NAcc Increased Ethanol Consumption and Preference}

Figure 2 shows ethanol consumption and preference for the NAcc GR7-scr $(n=10)$ and Gr7-shRNA $(n=10$ but one misplaced, $n=9$ ) injected rats. After recovery, these animals had access to four different ascending ethanol concentrations. During the daily ethanol consumption procedure, there was a significant effect of LV injection on ethanol consumption $\left(\mathrm{F}_{(1,68)}=9.254, p=0.003\right)$ as well as a significant effect of ethanol concentration $\left(\mathrm{F}_{(3,68)}=17.533\right.$, $p<0.001)$. More importantly, the interaction between LV injection and ethanol concentration was found as revealed 
by a two-way ANOVA with repeated measures. As shown in Figure 2a, simple main effect analysis showed that GR7shRNA-injected rats demonstrated an average increase of $40 \%$ in daily ethanol consumption relative to GR7-scrRNA controls at the 5, 10, and 20\% $v / v$ concentrations. A similar pattern was found for ethanol preference (Figure 2b). Again, a significant effect of lentiviral injection on ethanol preference was observed $\left(\mathrm{F}_{(1,68)}=43.322, p<0.001\right)$, as well as a significant interaction between $\mathrm{LV}$ injection and ethanol concentration $\left(\mathrm{F}_{(3,68)}=6.718, p<0.001\right)$. Ethanol preference was increased by an average of $60 \%$ in GR7shRNA-injected rats at the 10 and $20 \% \mathrm{v} / \mathrm{v}$ concentrations. More importantly, and as shown in Figure $2 c$, daily total fluid intake did not differ between the two groups during the continuous-access, two-bottle procedures $\left(\mathrm{F}_{(1,68)}=0.131\right.$, $p=0.718)$. In addition, no significant interaction was found between $\mathrm{LV}$ injection and ethanol concentration $\left(\mathrm{F}_{(3,68)}=\right.$ $0.320, p=0.811)$. One GR7-shRNA-injected rat did not show any change in ethanol consumption and preference and values were comparable to the control group, suggesting that lentiviral injections may have been most probably misplaced. This was confirmed later as no mGluR7 mRNA knockdown was detected in the NAcc in this rat (data not shown).

\section{GR7-shRNA and GR7-scr in the NAcc do not Show Differences in Taste Discrimination}

Since alterations in ethanol intake and preference can also result from differences in taste acceptability, the same rats were tested for saccharin (sweet) and quinine (bitter) taste consumptions and preferences using an order-balanced, two-bottle choice procedure. As depicted in Figure 3a and c, two-way ANOVAs with repeated measures identified significant effects of concentration for both saccharin $\left(\mathrm{F}_{(1,34)}=\right.$ 101.404, $p<0.001)$ and quinine consumption $\left(\mathrm{F}_{(1,34)}=494.477\right.$, $p<0.001)$ but no effect of lentiviral injection $\left(\left(\mathrm{F}_{(1,34)}=\right.\right.$ $0.016, p=0.902)$ and $\left(\mathrm{F}_{(1,34)}=0.164, p=0.688\right)$, respectively). More importantly, the interaction between LV injection and taste concentration was not significant (saccharin concentration $\times$ virus: $\left(F_{(1,34)}=0.022, p=0.883\right)$; quinine concentration $\times$ virus: $\left.\left(\mathrm{F}_{(1,34)}=0.083, p=0.775\right)\right)$. Also, and as depicted in Figure $3 b$ and $d$, there were no differences between GR7-scr- and GR7-shRNA-injected rats in total fluid intake for either saccharin or quinine during the tastant study $\left(\left(\mathrm{F}_{(1,34)}=0.018, p=0.894\right)\right.$ and $\left(\mathrm{F}_{(1,34)}=0.044\right.$, $p=0.834)$, respectively). More importantly, the interaction between LV injection and taste concentration was not significant (saccharin concentration $\times$ virus: $\left(\mathrm{F}_{(1,34)}=0.109\right.$,
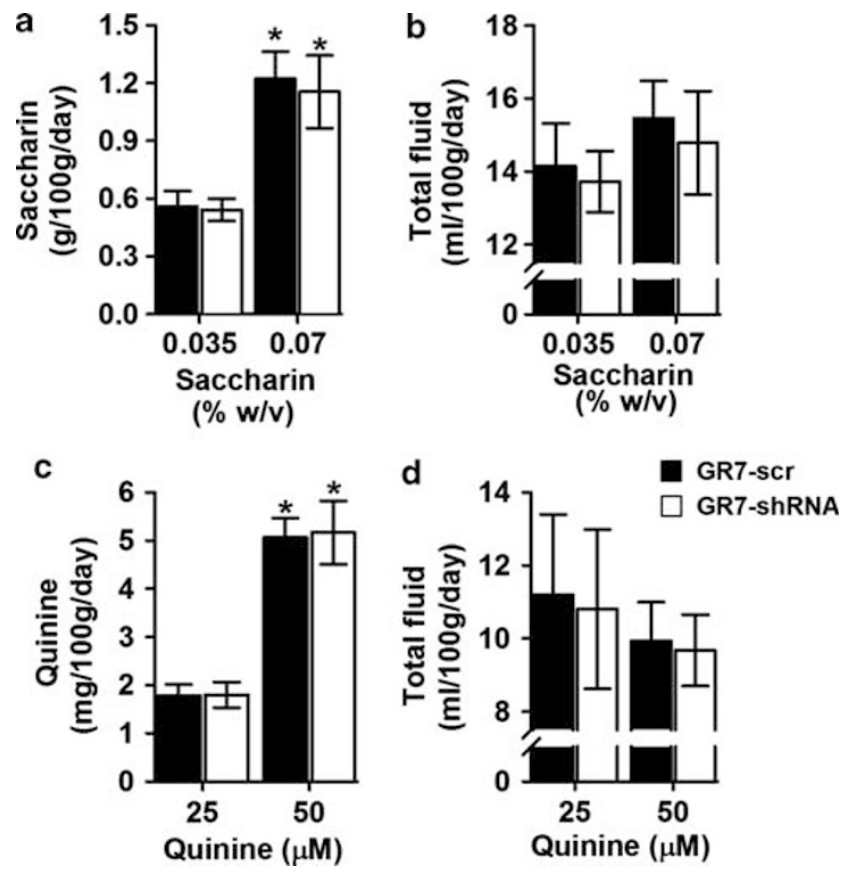

Figure 3 In the nucleus accumbens, GR7-scr and GR7-short hairpin RNA (shRNA)-injected rats showed similar consumption for the taste substances. (a) Saccharin consumption and (b) total fluid intake displayed by GR7-scr and GR7-shRNA rats are indicated. Saccharin consumption was expressed as a gram of saccharin solution consumed per $100 \mathrm{~g}$ of body weight. Total fluid intake was the sum of volume of the saccharin solution and water consumed per $100 \mathrm{~g}$ of body weight. (c) Quinine consumption and (d) total fluid intake displayed by GR7-scr and GR7-shRNA rats are shown. Quinine consumption was expressed as a milligram of quinine solution consumed per $100 \mathrm{~g}$ of body weight. Total fluid intake was the sum of the volume of quinine solution and water consumed per $100 \mathrm{~g}$ of body weight. Data are presented as the mean \pm SEM. $* 0<0.05$, the difference between the indicated group (two-way analysis of variance, Bonferroni's post hoc test).

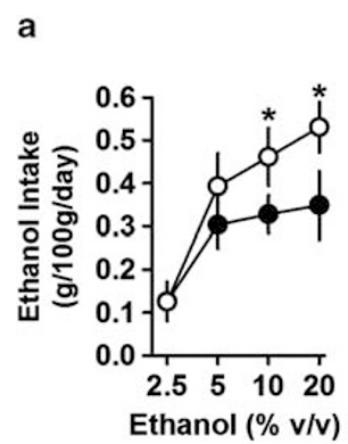

b $-\mathrm{GR7}$-scr OGR7-ShRNA

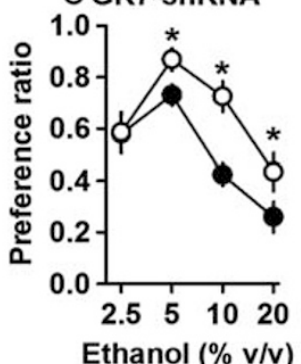

C

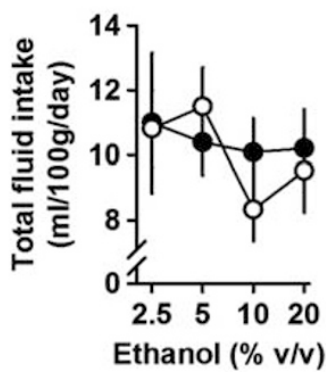

Figure 2 GR7-short hairpin RNA (shRNA) injection in the nucleus accumbens increased ethanol intake and ethanol preference in a two-bottle choice drinking paradigm. (a) Ethanol consumption and (b) ethanol preference displayed by GR7-scr and GR7-shRNA in the two-bottle free-choice paradigm test were plotted over increasing concentrations of ethanol. (a) GR7-shRNA rats consumed more ethanol than GR7-scr rats and pooled data are shown. (b) Ethanol preference was indicated as a ratio of ethanol volume to total fluid consumed. (c) Total fluid intake at the dose indicated. Data are presented as the mean \pm SEM. $* p<0.05$, the difference between the indicated group (two-way analysis of variance, Bonferroni's post hoc test). 
$p=0.743)$; quinine concentration $\times$ virus: $\left(\mathrm{F}_{(1,34)}=0.002\right.$, $p=0.968)$ ).

\section{GR7-shRNA in the DS did not Affect Ethanol Consumption and Preference}

Figure 4 shows ethanol consumption and preference for DS GR7-scr- $(n=8)$ and Gr7-shRNA $(n=8)$-injected rats with access to four different ethanol concentrations. GR7-scr and Gr7-shRNA rats showed similar consumption and preference for ethanol. As shown in Figure 4a, daily consumption for ethanol increased as concentrations of ethanol increased from 2.5 to $10 \%$ and declined at $20 \%$ (main effect of ethanol concentration: $\left.\mathrm{F}_{(3,56)}=18.552, p<0.001\right)$. A two-way ANOVA with repeated measures revealed that ethanol dose did not differ between the two groups (main effect of viral injection: $\mathrm{F}_{(1,56)}=1.669, p=0.202$; viral injection $\times$ ethanol concentration interaction: $\left.F_{(3,56)}=1.181, p=0.909\right)$. In addition, ethanol preference ratios also did not differ between GR7-scr and Gr7-shRNA groups (main effect of viral injection: $\mathrm{F}_{(1,56)}=0.272, p=0.604$; main effect of ethanol concentration: $\mathrm{F}_{(3,56)}=53.882, p<0.001$; viral injection $\times$ ethanol concentration interaction: $F_{(3,56)}=0.170$, $p=0.916$ ) (Figure $4 \mathrm{~b}$ ). Finally, and as depicted in Figure $4 \mathrm{c}$, daily total fluid intake did not differ between the two groups during the continuous-access, two-bottle procedures (main effect of viral injection: $\mathrm{F}_{(1,56)}=0.670, p=0.417$; main effect of ethanol concentration: $\mathrm{F}_{(3,56)}=0.599, p=0.618$; viral injection $\times$ ethanol concentration interaction: $\mathrm{F}_{(3,56)}=0.006$, $p=0.999)$.

\section{GR7-shRNA and GR7-scr in the DS do not Show Differences in Taste Sensitivity}

After 7 days of the ethanol intake study, the same rats were offered saccharin solution $v s$ water. Two-way ANOVArepeated measures followed by Bonferroni's post hoc test showed that saccharin consumption at $0.07 \%$ solution was increased compared with $0.035 \%$ solution in both GR7shRNA and GR7-scr rats (main effect of viral injection: $\mathrm{F}_{(1,28)}=0.383, p=0.541$; main effect of saccharin concentration: $\mathrm{F}_{(1,28)}=141.238, p<0.001$; viral injection $\times$ saccharin concentration interaction: $\left.\mathrm{F}_{(1,28)}=0.683, p=0.415\right)$ (Figure 5a).
There was no difference in the total fluid intake at each concentration tested between the two groups (main effect of viral injection: $\mathrm{F}_{(1,28)}=0.162, p=0.691$; main effect of saccharin concentration: $\mathrm{F}_{(1,28)}=8.801, p=0.006$; viral injection $\times$ saccharin concentration interaction: $F_{(1,28)}=$ $0.463, p=0.502$ ) (Figure 5b). After 7 days of the saccharin intake study, the rats were exposed to quinine solution $v \mathrm{~s}$ water. The consumption of quinine between the two groups was not different but there was an effect of quinine concentration (main effect of viral injection: $\mathrm{F}_{(1,28)}=$ $0.793, p=0.381$; main effect of saccharin concentration: $\mathrm{F}_{(1,28)}=71.991, p<0.001$; viral injection $\times$ saccharin concentration interaction: $\mathrm{F}_{(1,28)}=0.137, p=0.714$ ) (Figure $5 \mathrm{c}$ ). The fluid intake in GR7-shRNA- was similar to the GR7-scrinjected rats (main effect of viral injection: $\mathrm{F}_{(1,28)}=0.138$, $p=0.713$; main effect of quinine concentration: $\mathrm{F}_{(1,28)}=$ $0.456, p=0.505$; viral injection $\times$ saccharin concentration interaction: $\mathrm{F}_{(1,28)}=0.088, p=0.769$ ) (Figure $5 \mathrm{~d}$ ).

\section{In the NAcc, shRNA Injection Reduced mGluR7 mRNA Levels but had no Effect on BEC}

The stereotaxic microinjections of viral vectors were performed in the NAcc (Figure 6a). Real-time PCR analysis was performed to study the inhibitory effect of shRNA on mGluR7 mRNA levels in NAcc cDNA extracts of rats. GR7shRNA-treated rats exhibited a significant mean decrease of approximately $60 \%$ in total levels of mGluR7-mRNA compared with the GR7-scr control rats. A two-way ANOVA revealed that mRNA level differ brain between the two groups (main effect of viral injection: $\mathrm{F}_{(1,19)}=11.574, p=0.004$; main effect of region: $\left.\mathrm{F}_{(1,19)}=10.912, p=0.004\right)$. More importantly, the interaction between the virus and the region was significant $\left(\mathrm{F}_{(1,19)}=11.703, p=0.005\right)$ (Figure 6b). In contrast, when DS cDNA samples were used to quantify mGluR7 levels from the same rats, there was no significant difference between GR7-scr- and GR7-shRNA-injected rats $(p>0.05)$. We next tested the possibility that the higher sensitivity of GR7-shRNA rats to the rewarding properties of alcohol resulted from altered ethanol metabolism in the body. When blood was collected at 30,60,120,180, and $240 \mathrm{~min}$ after ethanol injection ( $2.5 \mathrm{~g} / \mathrm{kg}$ i.p.), it was found that the plasma ethanol levels were initially similar in both a

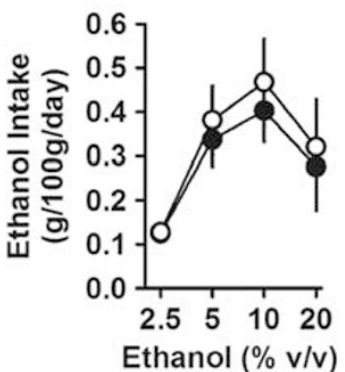

b

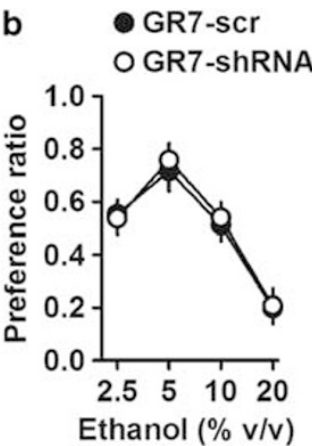

C

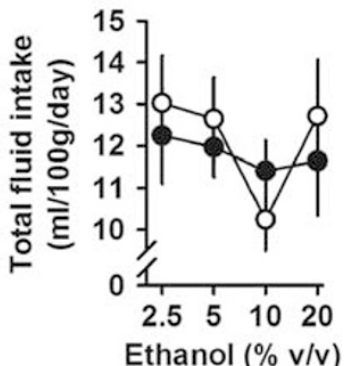

Figure 4 When injected into the dorsal striatum, GR7-scr and GR7-short hairpin RNA (shRNA) showed similar ethanol intake and ethanol preference in the test with a two-bottle free-choice paradigm. GR7-shRNA rats show normal voluntary ethanol consumption and preference. (a) Both groups showed a similar increase in ethanol consumption with increasing concentrations of ethanol up to $10 \% \mathrm{v} / \mathrm{v}$ ethanol. (b) Preference for ethanol over water and (c) total fluid intake were similar between GR7-scr and GR7-shRNA groups. Data are presented as the mean \pm SEM. $* p<0.05$, the difference between the indicated group (two-way analysis of variance, Bonferroni's post hoc test). 


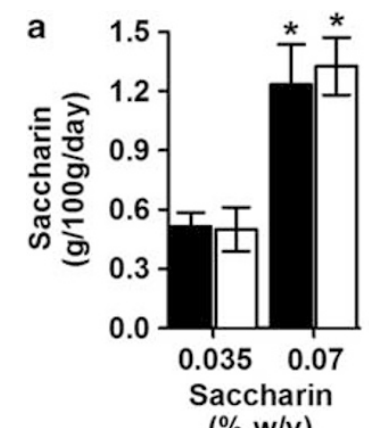

(\% w/v)

C

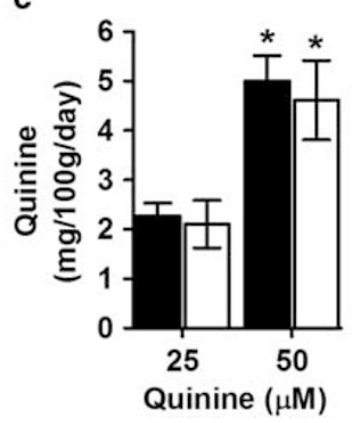

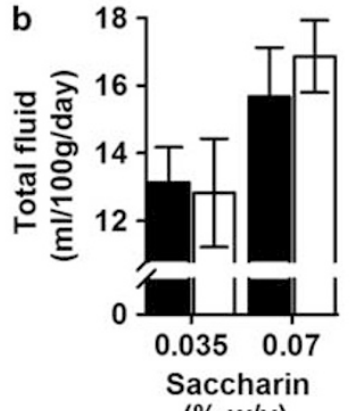

(\% w/v)

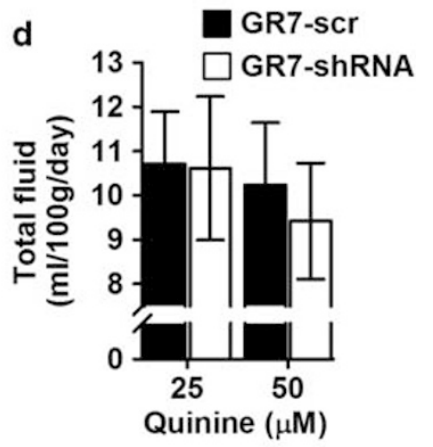

Figure 5 In the dorsal striatum, GR7-scr and GR7-short hairpin RNA (shRNA)-injected rats showed similar consumption for the taste substances. (a) Saccharin consumption and (b) total fluid intake displayed by GR7-scr and GR7-shRNA rats are indicated. Saccharin consumption was expressed as a gram of saccharin solution consumed per $100 \mathrm{~g}$ of body weight. Total fluid intake was the sum of the volume of saccharin solution and water consumed per $100 \mathrm{~g}$ of body weight. (c) Quinine consumption and (d) total fluid intake displayed by GR7-scr and GR7-shRNA rats are shown. Quinine consumption was expressed as a milligram of quinine solution consumed per $100 \mathrm{~g}$ of body weight. Total fluid intake was the sum of the volume of quinine solution and water consumed per $100 \mathrm{~g}$ of body weight. Data are presented as the mean \pm SEM. ${ }^{*} p<0.05$, the difference between the indicated group (two-way analysis of variance, Bonferroni's post hoc test).

groups and were also similarly decreased in a timedependent manner $\left(\mathrm{F}_{(4,28)}=18.354, p<0.001\right)$. In fact, the rate of ethanol metabolism as measured by the slope of the curves, was not significantly different between GR7-scr- and GR7-shRNA-injected rats (main effect of viral injection: $\left.\mathrm{F}_{(1,7)}=0.024, p=0.882\right)$. More importantly the interaction between viral injection and time was not significant $\left(\mathrm{F}_{(4,28)}=0.563, p=0.692\right)$ (Figure 6c).

\section{In the DS, shRNA Injection Reduced mGluR7 mRNA Levels but had no Effect on BEC}

The stereotaxic microinjections of viral vectors were aimed at the DS (Figure 7a). The quantification of mGluR7 mRNA using RT-PCR revealed that when GR7-shRNA was injected into the DS, transcript levels decreased to approximately $35 \%$ compared with the GR7-scr control rats. A two-way ANOVA revealed that mRNA level differ brain between the two groups (main effect of viral injection: $F_{(1,15)}=6.680$, $p=0.024$; main effect of region: $\left.\mathrm{F}_{(1,15)}=5.463, p=0.038\right)$. More importantly, the interaction between the virus and the region was significant $\left(\mathrm{F}_{(1,15)}=5.963, p=0.031\right)$ (Figure $7 \mathrm{~b}$ ). In the same rats, mGluR7 mRNA levels in the a LV injection in the NAcc
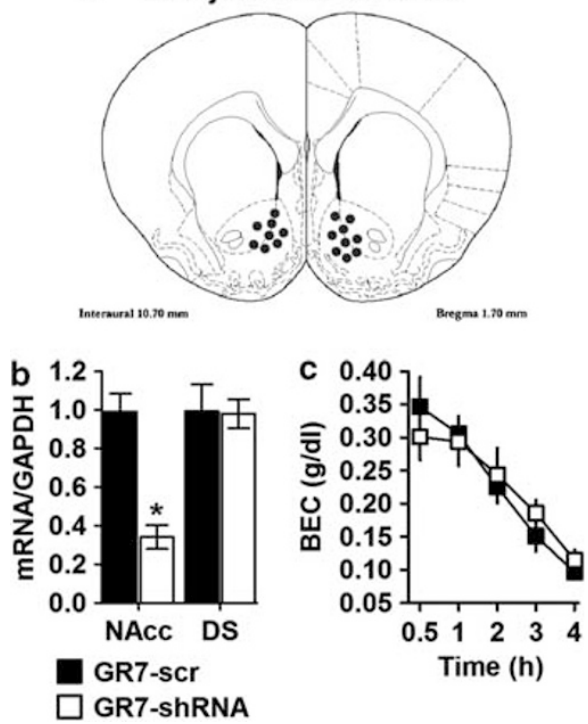

Figure 6 Lentiviral vector (LV) injection into the nucleus accumbens (NAcc), of GR7-short hairpin RNA (shRNA) but not GR7-scr, induces knockdown of mGluR7 mRNA expression in the NAcc. (a) Histological representation of injection placements in the NAcc. The distance anterior to bregma (in millimeters) is indicated. (b) Rats were stereotaxically microinjected with GR7-scr or GR7-shRNA into the NAcc. GR7-shRNA decreased mGluR7 mRNA expression in the NAcc but not in the dorsal striatum (DS). (c) Mean blood ethanol concentrations (BEC) of GR7-scr and GR7-shRNA injected rats into the NAcc following a single injection with ethanol $(2.5 \mathrm{~g} / \mathrm{kg}$ body weight, intraperitoneal). Blood was collected from the tail vein at various times after injection and analyzed to determine the ethanol concentration. Values represent the mean \pm SEM. ${ }^{*} p<0.01$, the difference between the indicated group (two-way analysis of variance, Bonferroni's post hoc test). GAPDH, glyceraldehyde-3-phosphate dehydrogenase.

NAcc were not affected by viral injections and no significant difference was found between GR7-scr and GR7-shRNAexpressing rats $(p>0.05)$. Blood alcohol levels changed across time following ethanol injection $(2.5 \mathrm{~g} / \mathrm{kg})\left(\mathrm{F}_{(4,24)}=\right.$ 20.433, $p<0.001)$. Figure $7 c$ shows that mGluR7 mRNA blockade in the DS did not significantly alter blood alcohol levels 30-240 min following ethanol administration (main effect of viral injection: $\mathrm{F}_{(1,6)}=0.149, p=0.712$; viral injection $\times$ time interaction: $\left.\mathrm{F}_{(4,24)}=0.316, p=0.865\right)$.

\section{DISCUSSION}

The present study investigated the role of accumbens mGluR7 in regulating ethanol behavioral output. We reported that infusion of GR7-shRNA into the NAcc resulted in robust knockdown of mGluR7 expression. In addition, NAcc mGluR7 mRNA blockade enhanced ethanolinduced CPP as well as alcohol consumption and preference in a two-bottle choice drinking paradigm. More importantly, mGluR7 mRNA knockdown in the DS affected neither ethanol-CPP nor voluntary alcohol drinking. Thus, in the present report, we combined behavioral and viral vector approaches to provide novel evidence that central glutamate signaling, through mGluR7, modulates ethanolinduced CPP and alcohol drinking in rats. 
a LV injection in the DS

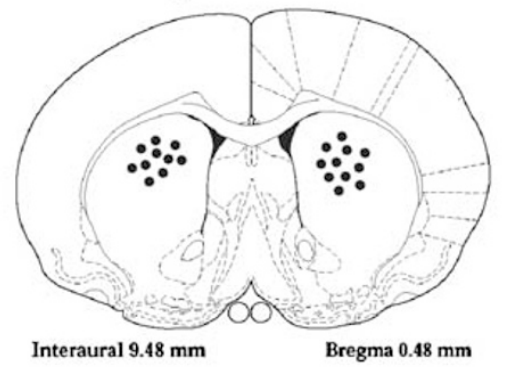

Interaural $9.48 \mathrm{~mm}$
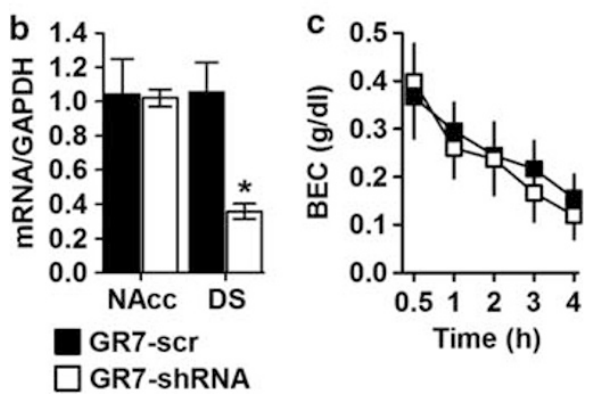

Figure 7 Lentiviral vector ( $L V$ ) injection into the dorsal striatum (DS), of GR7-short hairpin RNA (shRNA) but not GR7-scr, induces knockdown of mGluR7 mRNA expression in the DS. (a) Histological representation of injection placements in the DS. The distance anterior to bregma (in millimeters) is indicated. (b) Rats were stereotaxically microinjected with GR7-scr or GR7-shRNA into the DS. GR7-shRNA decreased mGluR7 mRNA expression in the DS but not in the nucleus accumbens (NAcc). (c) Mean blood ethanol concentrations (BEC) of GR7-scr- and GR7shRNA-injected rats into the DS following a single injection with ethanol $(2.5 \mathrm{~g} / \mathrm{kg})$. Blood was collected from the tail vein at various times after injection and analyzed to determine the ethanol concentration. Values represent the mean \pm SEM. $* p<0.001$, the difference between the indicated group (two-way analysis of variance, Bonferroni's post hoc test). GAPDH, glyceraldehyde-3-phosphate dehydrogenase.

Viral vectors were first validated in vitro. Using HEK293T cells co-transfected with GR7-shRNA and different plasmids expressing mGluR2, mGluR5, and mGluR7, we were able to show a knockdown of mGluR7 mRNA levels. In contrast mGluR2 and mGluR5 transcripts were not affected (see Supplementary Material). Taken together, these findings suggested that the prepared tools (GR7-shRNA) were adequate to manipulate mGluR7 expression in vivo. In the first experiment, we have shown that reduced expression of mGluR7 in NAcc neurons causes elevated CPP responses to ethanol. It has been shown that when infused into the NAcc, retro-transport of the virus from infected accumbal dopaminergic neurons back to the ventral tegmental area (VTA) was observed when the same viral vectors were used (Boyer and Dreyer, 2008). Consequently, rats that received NAcc injections of a viral vector encoding mGluR7-shRNA exhibited reduced mGluR7 mRNA levels in the VTA as well, suggesting viral uptake into the medium spiny projection neurons of the NAcc and translocation of shRNA from the soma of these neurons to their terminals in the VTA. Rats receiving these microinjections were more sensitive to the rewarding effects of ethanol. Considering that the enhanced preference to drug-paired environments and rewarding effects of psycho-stimulants appear to depend on common neural substrates (Green and Grahame, 2008; McBride et al,
1999; Risinger et al, 1994; Vlachou and Markou, 2010), these findings raised the possibility that reduced expression of mGlu7 receptors in the mesolimbic system would also affect the rewarding effects of ethanol. Indeed, lower doses of alcohol established-conditioned-place preferences in rats given this treatment, suggesting that these rats were more sensitive to the rewarding effects of the drug. Together, these findings suggest that mesolimbic mGluR7 has an important role in the regulation of the motivational effects of ethanol, presumably through their influence on the activity of VTA dopaminergic neurons. Using RT-PCR, we have shown that mGluR7 mRNA was decreased by up to $65 \%$ in the NAcc but not in the DS. In pilot studies, we observed that the scrambled vector produced results that were nearly identical to those in sham-injected animals (data not shown). Therefore, our demonstration that reduced expression of mGluR7 in the mesolimbic system enhances animal sensitivity to ethanol is not likely a result of nonspecific factors or off-target effects. Nevertheless these results are to be interpreted with caution. The possibility that a paucity of mGluR7 could lead to increased locomotor activity in general or in the presence of ethanol can't be excluded. Unfortunately, because of the technical reasons, locomotion was not recorded during the CPP experiment. In fact, possible high levels of locomotor activity in subjects experiencing low or absent levels of mGluR7 during testing could mask or reduce CPP effects. Conversely, reduced locomotor activity, in which subjects are traversing compartments (drug-seeking) to a lesser degree, could produce an erroneously high magnitude of place preference rendering potential interpretations limited.

It has also been shown that mGluR7 is involved in the rewarding properties of cocaine. In fact, the allosteric agonist AMN082, dose dependently inhibited cocaine-induced enhancement of electrical brain-stimulation and intravenous cocaine self-administration in rats (Li et al, 2009). In addition, microinjections of AMN082 into the NAcc or ventral pallidum, but not DS, also inhibited cocaine selfadministration in a dose-dependent manner (Li et al, 2009). The same group, has also shown that systemic and intraNAcc injection of AMN082, dose dependently inhibits cocaine-induced reinstatement of drug-seeking behavior (Li et al, 2010). This effect was not observed when AMN082 was infused into the DS. More importantly, MMPIP pretreatment reversed AMN082 effects on cocaine primed reinstatement ( $\mathrm{Li}$ et al, 2010). We have shown very recently that mGluR7 activation using AMN082 reduced ethanol-induced CPP reinstatement after extinction, an effect reversed by co-administration of the allosteric antagonist, MMPIP (Bahi, 2012). As for cocaine, these findings support our results that mGluR7 has a crucial role in addictive processes of ethanol as well.

There is considerable consensus that glutamate, via mGluR7, has a pivotal role in voluntary alcohol drinking. In fact, when $\mathrm{C} 57 \mathrm{BL} / 6 \mathrm{~J}$ male mice were trained to self-administer ethanol or sucrose, it was reported that AMN082 produced a significant reduction in ethanol and sucrose reinforced responding and inhibited locomotor activity (Salling et al, 2008). Vadasz et al (2007) when using near-isogenic advanced animal models with reduced genetic background interactions, were able to identify mGluR7 as a cis-regulated gene for ethanol consumption. The same group has reported 
recently that: first, mGluR7-knockout mice express increased ethanol consumption and preference. Second, subcongenic and congenic mice that overexpress mGluR7 mRNA consume less alcohol in a two-bottle choice drinking test (Gyetvai et al, 2011). Our previous work on ethanol voluntary intake in rats suggested that AMN082 $(10 \mathrm{mg} / \mathrm{kg})$ reduced ethanol consumption and preference, without affecting ethanol blood levels. This effect was blocked when MMPIP was co-administered with AMN082 (Bahi et al, 2012). Also, the current findings are consistent with our previous results where we have shown that mGluR7 activation, using AMN082, prevented development of ethanol dependence (Bahi, 2011). Also, ethanol-induced locomotor stimulation was blocked following mGluR7 activation and mice injected with AMN082 consumed less ethanol in a two-bottle free-choice paradigm and in a drinking-in-the-dark model. Impairment in reward mechanisms in AMN082-injected mice was confirmed by the lack of ethanol-CPP (Bahi, 2011). Our present results are consistent with the hypothesis that mGluR7 has a role in ethanol drinking and preference. Nevertheless, in our experiment, alcohol reference scores show that increasing ethanol concentrations were associated with decreased ethanol preference and reversal of preference in control rats, which is intriguing. mGluR7 knockdown did not change peak preference scores but instead seemed to reduce sensitivity to the deterrent effect of high concentrations of ethanol. One interpretation is that NAcc mGluR7 could modulate ethanol intake by reducing sensitivity to the aversive effects of high concentrations of ethanol.

As almost $90 \%$ of NAcc neurons are GABAergic medium spiny neurons (Brundege and Williams, 2002; Gerfen, 1988; Shi and Rayport, 1994) and because LV are not selective for any neuronal subtype, it is likely that the majority of the mGluR7-shRNA were expressed in these neurons. Furthermore, because we targeted the medial NAcc, the sub-region that projects predominantly to VTA, we believe that the effects we observed may involve mGlu7 receptors in the VTA. We interpret our results to suggest that ethanol-induced increase in extracellular glutamate can activate mGluR7 in NAcc projection fibers to the VTA (Carrara-Nascimento et al, 2011; Melendez et al, 2005; Piepponen et al, 2002; Xiao et al, 2009) This would enhance the sensitivity to the stimulant and rewarding effects of ethanol, although the possibility of relevant actions in other regions that receive projections from NAcc (eg, the ventral pallidum) (Hubert et al, 2011; Li et al, 2009; Maurice et al, 1998; Sharp et al, 2010) cannot be excluded. Both dopamine and glutamate levels increase significantly in the NAcc following ethanol administration (Di Chiara and Imperato, 1985, 1986; Imperato and Di Chiara, 1986; Siggins et al, 2003; Yan et al, 1998). Presumably this excess glutamate stimulates mGluRs in these brain regions. It is likely (although not yet demonstrated) that ethanol-induced glutamate activity participates in adaptive processes, such as those involved in sensitization, tolerance, withdrawal, and relapse (Bahi, 2011; Bahi et al, 2012; Salling et al, 2008). The receptor basis for these effects is poorly understood. However, in situ hybridization results suggest that several glutamate receptors may potentially be involved. These include mGluR1, 2, 3, 4, 5, and 7 (for example (Abe et al, 2001; Okamoto et al, 1994; Parelkar and Wang, 2004, 2008; Xi et al, 2003)). In summary, the present report confirms and extends accumulating evidence that mGluR7 seems to alter the sensitivity of brain reward circuits to ethanol.

In conclusion, the current data are consistent with the current literature indicating that voluntary ethanol intake can be mediated by glutamate signaling and that mGlu7 receptors have an important role in acquisition of ethanol drinking and CPP. The present results are consistent with pharmacological data indicating that the protective effects of AMN082 on ethanol drinking are mediated by mGluR7. From clinical data, a human genome-wide association study that was performed on subjects characterized for alcohol level of response phenotypes has identified glutamate signaling, and mGluR7 (3p26.1-p25.1) in alcohol use disorders (Joslyn et al, 2010). Thus, pharmacological tools aimed at activating mGluR7 might be helpful for preventing excessive drinking of alcohol. Studies using viral vectors to overexpress mGluR7 and examining ethanol-induced reinstatement of CPP or the effects of repeated withdrawal in dependent animals on alcohol consumption might further elucidate the roles of these receptors.

\section{ACKNOWLEDGEMENTS}

I thank Dr Julie Perroy (CNRS, Montpellier, France) and Dr Shigetada Nakanishi (Osaka Bioscience Institute, Osaka, Japan) for providing the mGluR7 and mGluR2 expressing vectors used for the in vitro studies. I acknowledge Dr JeanLuc Dreyer for the generous donation of the pTK431, $\mathrm{p} \Delta \mathrm{NRF}$, and pMDG-VSV used to clone and prepare mGluR7-shRNAexpressing lentiviral vectors. I thank Mr Mohamed Elwasila and Mr Mohamed Shafiullah for their technical assistance and Professor Keith Bagnall for his critical and careful proof reading.

\section{DISCLOSURE}

The author declares no conflict of interests. The author is receiving funds from the United Arab Emirates University (salary and research funding). The funder had no role in study design, data collection and analysis, decision to publish, or preparation of the manuscript.

\section{REFERENCES}

Abe S, Suzuki T, Ito T, Yamaguchi M, Baba A, Hori T et al (2001). Effects of single and repeated phencyclidine administration on the expression of metabotropic glutamate receptor subtype mRNAs in rat brain. Neuropsychopharmacology 25: 173-184.

Badanich KA, Adler KJ, Kirstein CL (2006). Adolescents differ from adults in cocaine conditioned place preference and cocaineinduced dopamine in the nucleus accumbens septi. Eur $J$ Pharmacol 550: 95-106.

Bahi A (2011). The pre-synaptic metabotropic glutamate receptor 7 'mGluR7' is a critical modulator of ethanol sensitivity in mice. Neuroscience 199: 13-23.

Bahi A (2012). The selective metabotropic glutamate receptor 7 allosteric agonist AMN082 prevents reinstatement of extinguished ethanol-induced conditioned place preference in mice. Pharmacol Biochem Behav 101: 193-200.

Bahi A, Boyer F, Bussard G, Dreyer JL (2005). Silencing dopamine D3-receptors in the nucleus accumbens shell in vivo induces changes in cocaine-induced hyperlocomotion. Eur J Neurosci 21: 3415-3426. 
Bahi A, Boyer F, Chandrasekar V, Dreyer JL (2008a). Role of accumbens BDNF and TrkB in cocaine-induced psychomotor sensitization, conditioned-place preference, and reinstatement in rats. Psychopharmacology 199: 169-182.

Bahi A, Boyer F, Gumy C, Kafri T, Dreyer JL (2004a). In vivo gene delivery of urokinase-type plasminogen activator with regulatable lentivirus induces behavioural changes in chronic cocaine administration. Eur J Neurosci 20: 3473-3488.

Bahi A, Boyer F, Kafri T, Dreyer JL (2004b). CD81-induced behavioural changes during chronic cocaine administration: in vivo gene delivery with regulatable lentivirus. Eur J Neurosci 19: 1621-1633.

Bahi A, Boyer F, Kafri T, Dreyer JL (2006). Silencing urokinase in the ventral tegmental area in vivo induces changes in cocaineinduced hyperlocomotion. J Neurochem 98: 1619-1631.

Bahi A, Dreyer JL (2008). Overexpression of plasminogen activators in the nucleus accumbens enhances cocaine-, amphetamine- and morphine-induced reward and behavioral sensitization. Genes Brain Behav 7: 244-256.

Bahi A, Dreyer JL (2012a). Involvement of nucleus accumbens dopamine D1 receptors in ethanol drinking, ethanol-induced conditioned place preference, and ethanol-induced psychomotor sensitization in mice. Psychopharmacology 222: 141-153.

Bahi A, Dreyer JL (2012b). Involvement of tissue plasminogen activator 'tPA' in ethanol-induced locomotor sensitization and conditioned-place preference. Behav Brain Res 226: 250-258.

Bahi A, Fizia K, Dietz M, Gasparini F, Flor PJ (2012). Pharmacological modulation of mGluR7 with AMN082 and MMPIP exerts specific influences on alcohol consumption and preference in rats. Addict Biol 17: 235-247.

Bahi A, Kusnecov A, Dreyer JL (2008b). The role of tissue-type plasminogen activator system in amphetamine-induced conditional place preference extinction and reinstatement. Neuropsychopharmacology 33: 2726-2734.

Bird MK, Lawrence AJ (2009). Group I metabotropic glutamate receptors: involvement in drug-seeking and drug-induced plasticity. Curr Mol Pharmacol 2: 83-94.

Bolonna AA, Kerwin RW, Munro J, Arranz MJ, Makoff AJ (2001). Polymorphisms in the genes for mGluR types 7 and 8: association studies with schizophrenia. Schizophr Res 47: 99-103.

Boyer F, Dreyer JL (2008). The role of gamma-synuclein in cocaine-induced behaviour in rats. Eur J Neurosci 27: 2938-2951.

Bradley SR, Uslaner JM, Flick RB, Lee A, Groover KM, Hutson PH (2011). The mGluR7 allosteric agonist AMN082 produces antidepressant-like effects by modulating glutamatergic signaling. Pharmacol Biochem Behav 101: 35-40.

Brundege JM, Williams JT (2002). Differential modulation of nucleus accumbens synapses. J Neurophysiol 88: 142-151.

Carrara-Nascimento PF, Griffin III WC, Pastrello DM, Olive MF, Camarini R (2011). Changes in extracellular levels of glutamate in the nucleus accumbens after ethanol-induced behavioral sensitization in adolescent and adult mice. Alcohol 45: 451-460.

Cryan JF, Kelly PH, Neijt HC, Sansig G, Flor PJ, van Der Putten H (2003). Antidepressant and anxiolytic-like effects in mice lacking the group III metabotropic glutamate receptor mGluR7. Eur J Neurosci 17: 2409-2417.

Dackis CA, O'Brien CP (2001). Cocaine dependence: a disease of the brain's reward centers. J Subst Abuse Treat 21: 111-117.

De Witte P, Littleton J, Parot P, Koob G (2005). Neuroprotective and abstinence-promoting effects of acamprosate: elucidating the mechanism of action. CNS Drugs 19: 517-537.

Di Chiara G, Imperato A (1985). Ethanol preferentially stimulates dopamine release in the nucleus accumbens of freely moving rats. Eur J Pharmacol 115: 131-132.

Di Chiara G, Imperato A (1986). Preferential stimulation of dopamine release in the nucleus accumbens by opiates, alcohol, and barbiturates: studies with transcerebral dialysis in freely moving rats. Ann N Y Acad Sci 473: 367-381.
Di Chiara G, Tanda G, Frau R, Carboni E (1993). On the preferential release of dopamine in the nucleus accumbens by amphetamine: further evidence obtained by vertically implanted concentric dialysis probes. Psychopharmacology 112: 398-402.

Fendt M, Schmid S, Thakker DR, Jacobson LH, Yamamoto R, Mitsukawa $\mathrm{K}$ et al (2008). mGluR7 facilitates extinction of aversive memories and controls amygdala plasticity. Mol Psychiatry 13: 970-979.

Flatscher-Bader T, Zuvela N, Landis N, Wilce PA (2008). Smoking and alcoholism target genes associated with plasticity and glutamate transmission in the human ventral tegmental area. Hum Mol Genet 17: 38-51.

Gerfen CR (1988). Synaptic organization of the striatum. J Electron Microsc Tech 10: 265-281.

Green AS, Grahame NJ (2008). Ethanol drinking in rodents: is freechoice drinking related to the reinforcing effects of ethanol? Alcohol 42: 1-11.

Gyetvai B, Simonyi A, Oros M, Saito M, Smiley J, Vadasz C (2011). mGluR7 genetics and alcohol: intersection yields clues for addiction. Neurochem Res 36: 1087-1100.

Hubert GW, Manvich DF, Kuhar MJ (2011). Cocaine and amphetamine-regulated transcript-containing neurons in the nucleus accumbens project to the ventral pallidum in the rat and may inhibit cocaine-induced locomotion. Neuroscience 165: 179-187.

Imperato A, Di Chiara G (1986). Preferential stimulation of dopamine release in the nucleus accumbens of freely moving rats by ethanol. J Pharmacol Exp Ther 239: 219-228.

Joslyn G, Ravindranathan A, Brush G, Schuckit M, White RL (2010). Human variation in alcohol response is influenced by variation in neuronal signaling genes. Alcohol Clin Exp Res 34: 800-812.

Keiver K, Duggal S, Simpson ME (2005). Ethanol administration results in a prolonged decrease in blood ionized calcium levels in the rat. Alcohol 37: 173-178.

Kemppainen H, Raivio N, Nurmi H, Kiianmaa K (2010). GABA and glutamate overflow in the VTA and ventral pallidum of alcoholpreferring AA and alcohol-avoiding ANA rats after ethanol. Alcohol Alcohol 45: 111-118.

Kinoshita A, Shigemoto R, Ohishi H, van der Putten H, Mizuno N (1998). Immunohistochemical localization of metabotropic glutamate receptors, mGluR7a and mGluR7b, in the central nervous system of the adult rat and mouse: a light and electron microscopic study. J Comp Neurol 393: 332-352.

Li X, Li J, Gardner EL, Xi ZX (2010). Activation of mGluR7s inhibits cocaine-induced reinstatement of drug-seeking behavior by a nucleus accumbens glutamate-mGluR2/3 mechanism in rats. J Neurochem 114: 1368-1380.

Li X, Li J, Peng XQ, Spiller K, Gardner EL, Xi ZX (2009). Metabotropic glutamate receptor 7 modulates the rewarding effects of cocaine in rats: involvement of a ventral pallidal GABAergic mechanism. Neuropsychopharmacology 34: 1783-1796.

Li Y, Kauer JA (2004). Repeated exposure to amphetamine disrupts dopaminergic modulation of excitatory synaptic plasticity and neurotransmission in nucleus accumbens. Synapse 51: 1-10.

Maurice N, Deniau JM, Glowinski J, Thierry AM (1998). Relationships between the prefrontal cortex and the basal ganglia in the rat: physiology of the corticosubthalamic circuits. J Neurosci 18: 9539-9546.

McBride WJ, Murphy JM, Ikemoto S (1999). Localization of brain reinforcement mechanisms: intracranial self-administration and intracranial place-conditioning studies. Behav Brain Res 101: $129-152$.

Melendez RI, Hicks MP, Cagle SS, Kalivas PW (2005). Ethanol exposure decreases glutamate uptake in the nucleus accumbens. Alcohol Clin Exp Res 29: 326-333.

Mitsukawa K, Mombereau C, Lotscher E, Uzunov DP, van der Putten H, Flor PJ et al (2006). Metabotropic glutamate receptor subtype 7 ablation causes dysregulation of the HPA axis and 
increases hippocampal BDNF protein levels: implications for stress-related psychiatric disorders. Neuropsychopharmacology 31: 1112-1122.

O'Connor RM, Finger BC, Flor PJ, Cryan JF (2010). Metabotropic glutamate receptor 7: at the interface of cognition and emotion. Eur J Pharmacol 639: 123-131.

Ohtsuki T, Koga M, Ishiguro H, Horiuchi Y, Arai M, Niizato K et al (2008). A polymorphism of the metabotropic glutamate receptor mGluR7 (GRM7) gene is associated with schizophrenia. Schizophr Res 101: 9-16.

Okamoto N, Hori S, Akazawa C, Hayashi Y, Shigemoto R, Mizuno $\mathrm{N}$ et al (1994). Molecular characterization of a new metabotropic glutamate receptor mGluR7 coupled to inhibitory cyclic AMP signal transduction. J Biol Chem 269: 1231-1236.

Olive MF (2009). Metabotropic glutamate receptor ligands as potential therapeutics for addiction. Curr Drug Abuse Rev 2: 83-98.

Parelkar NK, Wang JQ (2004). mGluR5-dependent increases in immediate early gene expression in the rat striatum following acute administration of amphetamine. Brain Res Mol Brain Res 122: $151-157$.

Parelkar NK, Wang JQ (2008). Upregulation of metabotropic glutamate receptor $8 \mathrm{mRNA}$ expression in the rat forebrain after repeated amphetamine administration. Neurosci Lett 433: 250-254.

Paxinos G, Watson C (1998). The Rat Brain in Stereotaxic Coordinates. Academic Press: San Diego.

Pelc I (1997). [Alcoholism: relapse prevention]. Rev Med Brux 18: 272-276.

Piepponen TP, Kiianmaa K, Ahtee L (2002). Effects of ethanol on the accumbal output of dopamine, GABA and glutamate in alcohol-tolerant and alcohol-nontolerant rats. Pharmacol Biochem Behav 74: 21-30.

Risinger FO, Malott DH, Prather LK, Niehus DR, Cunningham CL (1994). Motivational properties of ethanol in mice selectively bred for ethanol-induced locomotor differences. Psychopharmacology 116: 207-216.

Salling MC, Faccidomo S, Hodge CW (2008). Nonselective suppression of operant ethanol and sucrose self-administration by the mGluR7 positive allosteric modulator AMN082. Pharmacol Biochem Behav 91: 14-20.

Sharp BM, Chen H, Gong S, Wu X, Liu Z, Hiler K et al (2010). Gene expression in accumbens GABA neurons from inbred rats with different drug-taking behavior. Genes Brain Behav 10: 778-788.

Shi WX, Rayport S (1994). GABA synapses formed in vitro by local axon collaterals of nucleus accumbens neurons. J Neurosci 14: $4548-4560$.

Siggins GR, Martin G, Roberto M, Nie Z, Madamba S, De Lecea L (2003). Glutamatergic transmission in opiate and alcohol dependence. Ann N Y Acad Sci 1003: 196-211.

Uys JD, LaLumiere RT (2008). Glutamate: the new frontier in pharmacotherapy for cocaine addiction. CNS Neurol Disord Drug Targets 7: 482-491.

Vadasz C, Saito M, Gyetvai BM, Oros M, Szakall I, Kovacs KM et al (2007). Glutamate receptor metabotropic 7 is cis-regulated in the mouse brain and modulates alcohol drinking. Genomics 90: 690-702.

Vlachou S, Markou A (2010). GABAB receptors in reward processes. Adv Pharmacol 58: 315-371.

Wenger T, Moldrich G, Furst S (2003). Neuromorphological background of cannabis addiction. Brain Res Bull 61: 125-128.

Wills TA, Klug JR, Silberman Y, Baucum AJ, Weitlauf C, Colbran $\mathrm{RJ}$ et al (2012). GluN2B subunit deletion reveals key role in acute and chronic ethanol sensitivity of glutamate synapses in bed nucleus of the stria terminalis. Proc Natl Acad Sci USA 109: E278-287.

Xi ZX, Shen H, Baker DA, Kalivas PW (2003). Inhibition of nonvesicular glutamate release by group III metabotropic glutamate receptors in the nucleus accumbens. J Neurochem 87: 1204-1212.

Xiao C, Shao XM, Olive MF, Griffin III WC, Li KY, Krnjevic K et al (2009). Ethanol facilitates glutamatergic transmission to dopamine neurons in the ventral tegmental area. Neuropsychopharmacology 34: 307-318.

Yan QS, Reith ME, Yan SG, Jobe PC (1998). Effect of systemic ethanol on basal and stimulated glutamate releases in the nucleus accumbens of freely moving Sprague-Dawley rats: a microdialysis study. Neurosci Lett 258: 29-32.

Supplementary Information accompanies the paper on the Neuropsychopharmacology website (http://www.nature.com/npp) 